\title{
Concerns About Ring and Rosing's Omega Project
}

To the Editor:

While I have long been an admirer of the work of Kenneth Ring, I found Ring and Christopher Rosing's (1990) recent article, "The Omega Project: An Empirical Study of the NDE-Prone Personality," troublesome for several reasons.

The weakest part of this article was the Instruments section. Of the nine tests reported, only three have been cited in other studies and all to date are unpublished. While this is not a problem in and of itself, no data on norms, reliability, or validity of these tests are given, with the exception of incomplete data on the Psychological Inventory. While it is customary to omit this information on well-known tests like the 
Minnesota Multiphasic Personality Inventory (MMPI), this lack of basic information seriously weakens the study.

In a study that has as its stated purpose exploring the personality of the near-death experiencer (NDEr), it would have been nice to have one or two major personality tests, such as the Personal Orientation Inventory, which measures mental health, the Loevinger Test of Ego Development, which measures global ego development, and/or the MMPI or similar test of psychopathology. I mention the latter since the authors raised the question of dissociative disorders among NDErs.

Additionally, in spite of their thoughtful disclaimer to the contrary in the Implications and Conclusions section of their paper, it cannot be stressed enough that the NDE is independent of psychopathology. In this regard I offer the following points:

1. NDEs can happen to anyone; no one plans a heart attack or a death on the operating table, and only the suicidal plan car wrecks, drownings, or other life threatening events;

2. while figures vary from study to study, 27 to 62 percent of individuals near death report NDEs (Rogo, 1989); and

3. the number of NDErs is quite a bit higher than population estimates of dissociative disorders (National Institute of Mental Health, 1985).

In other words, some NDErs may have emotional problems and may have been abused, but it is unlikely that most have. It is noteworthy that among mental health patient populations, victims of childhood sexual abuse often show signs of dissociative disorders (Gregory-Bills, 1988), but among normal, nonclinical samples, victims of childhood sexual abuse do not show retarded development (Jennings, 1989). The difference is one of sampling.

There are differences between NDEs and related paranormal and abnormal states. In discussing this subject in classes on abnormal human behavior, I have pointed out that NDEs differ from out-of-body experiences (OBEs) in that they include features common to all subjects while OBEs are unique to each person, and NDEs are involuntary while OBEs are voluntary; and NDEs differ from dissociative disorders in that they again share universal features while dissociative disorders are unique to each person, and NDEs are usually pleasurable while dissociative disorders are characterized by marked distress.

I continue to admire Ring's work and hope my overall comments have been helpful and not hurtful. 


\section{References}

Gregory-Bills, T. (1988). A comparison of therapy outpatients with intra-family and extrafamily sexual abuse and patients without sexual abuse. Unpublished doctoral dissertation, University of Houston, Houston.

Jennings, A.G. (1989). Ego development in sexually abused women. Unpublished doctoral dissertation, University of Houston, Houston.

National Institute of Mental Health. (1985). Mental health in the United States, 1985. Washington, DC: U.S. Government Printing Office.

Ring, K., and Rosing, C.J. (1990). The Omega Project: An empirical study of the NDEprone personality. Journal of Near-Death Studies, 8, 211-229.

Rogo, D.S. (1989). Return from silence: $A$ study of near-death experiences. Wellingborough, England: Aquarian Press.

Ken R. Vincent, Ed.D. 12463 Kimberley Houston, TX 77024 